\title{
ON A DISCRETE BOLTZMANN-SMOLUCHOWSKI EQUATION WITH RATES BOUNDED IN THE VELOCITY VARIABLES
}

\author{
NICOLAS FOURNIER * AND STÉPHANE MISCHLER ${ }^{\dagger}$
}

\begin{abstract}
Consider a spatially homogeneous infinite particle system in which coalescence and elastic collisions occur. The Boltzmann-Smoluchowski equation describes the evolution of the concentration $f(t, m, v)$ of particles of mass $m$ and velocity $v$ at time $t \geq 0$. Using a stochastic version of this equation, we give an exact simulation scheme and we study the asymptotics of solutions for large times.
\end{abstract}

Key words. Boltzmann equations, Smoluchowski equations, Jump processes.

MSC $2000: 82 \mathrm{C} 40,60 \mathrm{~J} 75$.

\section{Introduction}

We consider a system of particles characterized by their mass $m \in(0, \infty)$ and velocity $v \in \mathbb{R}^{3}$. We assume that two particles of characteristics $(m, v)$ and $\left(m_{*}, v_{*}\right)$ may coalesce to give a larger particle at rate $a_{S}\left(m, m_{*}\right) \varphi_{S}\left(v, v_{*}\right)$, or they may collide to give two particles with different velocities at rate $a_{B}\left(m, m_{*}\right) \varphi_{B}\left(v, v_{*}\right)$. Then the Boltzmann-Smoluchowski equation $(B S)$ (see Definition 2.1) describes the evolution of the concentration density $f(t, m, v)$ of particles of mass $m$ and velocity $v$ at time $t \geq 0$.

Collisional invariants have been described by Hylkema-Villedieu [11], and the pure kinetic coalescence equation $\left(a_{B} \varphi_{B} \equiv 0\right)$ has been studied by Roquejoffre-Villedieu [8] and Escobedo-Laurençot-Mischler [4] .

From a physical point of view, particles evolving according to these rules are met, for instance, in dense sprays of liquid droplets, see Hylkema-Villedieu [11], Baranger [1], and the reference therein for a desciption of models. They are also met in astrophysics (in order to describe formation of galaxies) and we refer to Bobylev-Illner [2] for mathematical models in this context.

Typical rates (in the hard spheres case) are $a_{S}\left(m, m_{*}\right)=a_{B}\left(m, m_{*}\right)=\left(m^{1 / 3}+\right.$ $\left.m_{*}^{1 / 3}\right)^{2}, \varphi_{S}\left(v, v_{*}\right)=\varphi_{B}\left(v, v_{*}\right)=\left|v-v_{*}\right|$. We will deal with general assumptions, which contain this case, in a work in preparation [6]. However, we consider in the present paper the simpler case where $\varphi_{S}$ and $\varphi_{B}$ are bounded, while the masses of the particles belong to $\mathbb{N}^{*}$ (discrete case).

To study equation $(B S)$, we introduce a pure jump stochastic Markov process $\left(M_{t}, V_{t}\right)_{t \geq 0}$, which shall be seen as the evolution of the characteristics (mass, velocity) of a typical particle. More precisely, the link between this process and equation $(B S)$ is the following: the function

$$
f(t, m, v)=m^{-1} P\left[M_{t}=m, V_{t} \in d v\right]
$$

satisfies $(B S)$. The stochastic process contains however more information than the deterministic equation $(B S)$, since it contains historic information on typical particles. We refer to Tanaka [10], Sznitman [9], Graham-Méléard [7] for such a technique for

\footnotetext{
${ }^{*}$ Institut Elie Cartan, Campus Scientifique, BP 239, 54506, Vandoeuvre-lès-Nancy Cedex, France (fournier@iecn.u-nancy.fr).

${ }^{\dagger}$ Département de Mathématiques, Université de Versailles-Saint-Quentin, Bâtiment Fermat, 45, avenue des Etats-Unis, 78055, Versailles Cedex, France (mischler@math.uvsq.fr); Projet BANG, INRIA Rocquencourt B.P.105 78153, Le Chesnay CEDEX, France.
} 
the Boltzmann equation, and to Deaconu-Fournier-Tanré [3] for the Smoluchowski equation.

Our main aim here is to use the stochastic interpretation of $(B S)$ :

1) to derive an exact simulation scheme of the process $\left(M_{t}, V_{t}\right)$ which yields an immediate and constructive existence proof for $(B S)$.

2 ) to show that as $t$ tends to infinity, $M_{t}$ tends almost surely to infinity, which in particular implies that the solution $f(t,$.$) to (B S)$ tends to 0 in $L^{1}$. Although the probabilistic proof is quite easy, we are not able, for the moment, to handle a deterministic proof (which has been done in [4] in the case without collisions $a_{B} \varphi_{B} \equiv 0$ ).

The paper is organized as follows: in Section 2, we give definitions of equation $(B S)$ and of typical particles. We also state our results. In Section 3, we show how to simulate exactly the typical particles. Finally, Section 4 is devoted to the large time behavior.

\section{Notations and results}

We denote by $S_{2}$ the sphere of $\mathbb{R}^{3}$, which will be used to model the impact parameters of the collisions. Let us first introduce our hypotheses.

Assumption $(A)$ : There exists a constant $A$ such that:

1. The two maps $a_{S}$ and $a_{B}$ from $\mathbb{N}^{*} \times \mathbb{N}^{*}$ into $\mathbb{R}_{+}$satisfy, for all $m, m_{*}$ in $\mathbb{N}^{*}$

$$
\begin{gathered}
a_{S}\left(m, m_{*}\right)=a_{S}\left(m_{*}, m\right) \leq A\left(m+m_{*}\right) \\
a_{B}\left(m, m_{*}\right)=a_{B}\left(m_{*}, m\right) \leq A\left(m+m_{*}\right) .
\end{gathered}
$$

2. The maps $\varphi_{S}$ from $\mathbb{R}^{3} \times \mathbb{R}^{3}$ into $\mathbb{R}_{+}$and $\varphi_{B}$ from $\mathbb{R}^{3} \times \mathbb{R}^{3} \times S_{2}$ into $\mathbb{R}_{+}$satisfy, for all $v, v_{*}$ in $\mathbb{R}^{3}$, all $n \in S_{2}$,

$$
\varphi_{S}\left(v, v_{*}\right)=\varphi_{S}\left(v_{*}, v\right) \leq A ; \quad \varphi_{B}\left(v, v_{*}, n\right)=\varphi_{B}\left(v_{*}, v, n\right) \leq A
$$

3. The initial condition $f_{0}$ from $\mathbb{N}^{*} \times \mathbb{R}^{3}$ into $\mathbb{R}_{+}$satisfies

$$
\sum_{m \geq 1} \int_{\mathbb{R}^{3}} m f_{0}(m, v) d v=1 ; \quad \sum_{m \geq 1} \int_{\mathbb{R}^{3}}\left(m^{2}+m|v|^{2}\right) f_{0}(m, v) d v<\infty
$$

For $(m, v)$ and $\left(m_{*}, v_{*}\right)$ in $\mathbb{N}^{*} \times \mathbb{R}^{3}$, we denote by $\left(m_{* *}, v_{* *}\right)$ the post-coagulation characteristics, given by

$$
m_{* *}=m+m_{*} ; \quad v_{* *}=\frac{m v+m_{*} v_{*}}{m+m_{*}} .
$$

Note that in a coalescence, the mass $m$ and momentum $m v$ are preserved, while the kinetic energy decreases: $m_{* *}\left|v_{* *}\right|^{2}=m|v|^{2}+m_{*}\left|v_{*}\right|^{2}-\frac{m m_{*}}{m+m_{*}}\left|v-v_{*}\right|^{2}$. We denote by $L_{S}$ the associated operator acting on bounded measurable functions $\phi$ from $\mathbb{N}^{*} \times \mathbb{R}^{3}$ into $\mathbb{R}$ by

$$
\begin{array}{r}
L_{S} \phi\left[(m, v),\left(m_{*}, v_{*}\right)\right]=\frac{1}{2}\left\{\phi\left(m_{* *}, v_{* *}\right)-\phi(m, v)-\phi\left(m_{*}, v_{*}\right)\right\} \\
a_{S}\left(m, m_{*}\right) \varphi_{S}\left(v, v_{*}\right) .
\end{array}
$$


For $(m, v)$ and $\left(m_{*}, v_{*}\right)$ in $\mathbb{N}^{*} \times \mathbb{R}^{3}$ and $n \in S_{2}$, we denote by $\left(m^{\prime}, v^{\prime}\right)$ and $\left(m_{*}^{\prime}, v_{*}^{\prime}\right)$ the post-collision characteristics given by

$$
\begin{array}{r}
m^{\prime}=m ; \quad v^{\prime}=v+2 \frac{m_{*}}{m+m_{*}}\left[\left(v-v_{*}\right) \cdot n\right] n \\
m_{*}^{\prime}=m_{*} ; \quad v_{*}^{\prime}=v_{*}-2 \frac{m}{m+m_{*}}\left[\left(v-v_{*}\right) \cdot n\right] n .
\end{array}
$$

Collisions thus preserve mass, momentum, and kinetic energy. Then, for $\phi$ bounded and measurable from $\mathbb{N}^{*} \times \mathbb{R}^{3}$ into $\mathbb{R}$, we set

$$
\begin{gathered}
L_{B} \phi\left[(m, v),\left(m_{*}, v_{*}\right)\right]=\frac{1}{2} \int_{S_{2}}\left\{\phi\left(m^{\prime}, v^{\prime}\right)+\phi\left(m_{*}^{\prime}, v_{*}^{\prime}\right)-\phi(m, v)-\phi\left(m_{*}, v_{*}\right)\right\} \\
a_{B}\left(m, m_{*}\right) \varphi_{B}\left(v, v_{*}, n\right) d n \\
=\int_{S_{2}}\left\{\phi\left(m^{\prime}, v^{\prime}\right)-\phi(m, v)\right\} a_{B}\left(m, m_{*}\right) \varphi_{B}\left(v, v_{*}, n\right) d n
\end{gathered}
$$

the second equality being a consequence of our symmetry assumptions. We now may define equation $(B S)$.

Definition 2.1. A function $f$ from $[0, \infty) \times \mathbb{N}^{*} \times \mathbb{R}^{3}$ into $\mathbb{R}_{+}$is said to be a solution to $(B S)$ if:

(i) for all $t \geq 0, \sum_{m \geq 1} \int_{\mathbb{R}^{3}} m f(t, m, v) d v=1$,

(ii) for all bounded measurable function $\phi$ from $\mathbb{N}^{*} \times \mathbb{R}^{3}$ into $\mathbb{R}$, for all $t \geq 0$,

$$
\begin{array}{r}
\sum_{m \geq 1} \int_{\mathbb{R}^{3}} \phi(m, v) f(t, m, v) d v=\sum_{m \geq 1} \int_{\mathbb{R}^{3}} \phi(m, v) f_{0}(m, v) d v \\
+\int_{0}^{t} d s \sum_{m \geq 1} \int_{\mathbb{R}^{3}} d v \sum_{m_{*} \geq 1} \int_{\mathbb{R}^{3}} d v_{*}\left\{L_{S} \phi+L_{B} \phi\right\}\left[(m, v),\left(m_{*}, v_{*}\right)\right] \\
f(s, m, v) f\left(s, m_{*}, v_{*}\right) .
\end{array}
$$

This equation is quite natural. For example, the term containing $L_{S}$ explains that there is coalescence between particles of characteristics $(m, v)$ and $\left(m_{*}, v_{*}\right)$, at rate $a_{S}\left(m, m_{*}\right) \varphi_{S}\left(v, v_{*}\right)$, and proportionally to the concentrations $f(t, m, v)$ and $f\left(t, m_{*}, v_{*}\right)$. Note that Assumption $(A)$ and condition (i) ensures that everything makes sense in (ii).

We now introduce a stochastic version of this equation, that contains more information about the particles, which will be useful to study the large time behavior of solutions.

Definition 2.2. Assume $(A)$. Let $\left(\Omega, \mathcal{F},\left(\mathcal{F}_{t}\right)_{t \geq 0}, P\right)$ be a (sufficiently large) probability space. A stochastic process $\left(M_{t}, V_{t}\right)_{t \geq 0}$ is a solution to $(S D E)$ if the following conditions hold.

(a) $M$ is a càdlàg adapted nondecreasing $\mathbb{N}^{*}$-valued process, while $V$ is a càdlàg adapted $\mathbb{R}^{3}$-valued process.

(b) The law of $\left(M_{0}, V_{0}\right)$ is given by $\sum_{k \geq 1} k f_{0}(k, v) \delta_{k}(d m) d v$.

(c) For all $T<\infty, E\left[M_{T}+\left|V_{T}\right|^{2}\right]<\infty$.

(d) Denote, for each $t \geq 0$, by $Q_{t}$ the law of $\left(M_{t}, V_{t}\right)$ (it is a probability measure on $\left.\mathbb{N}^{*} \times \mathbb{R}^{3}\right)$. There exist two independent $\left(\mathcal{F}_{t}\right)_{t>0}$-adapted Poisson measures $\nu_{S}(d s, d(m, v), d u)$ on $[0, \infty) \times\left(\mathbb{N}^{*} \times \mathbb{R}^{3}\right) \times[0, \infty)$ and $\nu_{B}(\bar{d} s, d(m, v), d n, d u)$ on $[0, \infty) \times$ 
$\left(\mathbb{N}^{*} \times \mathbb{R}^{3}\right) \times S_{2} \times[0, \infty)$ with intensity measures $d s Q_{s}(d m, d v) d u$ and $d s Q_{s}(d m, d v) d n d u$ such that a.s., for all $t \geq 0$,

$$
\begin{gathered}
M_{t}=M_{0}+\int_{0}^{t} \int_{\mathbb{N}^{*} \times \mathbb{R}^{3}} \int_{0}^{\infty} m \mathbb{1}_{\left\{u \leq \frac{a_{S}\left(M_{s-}, m\right)}{m} \varphi_{S}\left(V_{s-}, v\right)\right\}} \nu_{S}(d s, d(m, v), d u), \\
V_{t}=V_{0}+\int_{0}^{t} \int_{\mathbb{N}^{*} \times \mathbb{R}^{3}} \int_{0}^{\infty} \frac{m\left(v-V_{s-}\right)}{m+M_{s-}} \mathbb{1}_{\left\{u \leq \frac{a_{S}\left(M_{s-}, m\right)}{m} \varphi_{S}\left(V_{s-}, v\right)\right\}} \nu_{S}(d s, d(m, v), d u) \\
+\int_{0}^{t} \int_{\mathbb{N}^{*} \times \mathbb{R}^{3}} \int_{S_{2}} \int_{0}^{\infty} 2 \frac{m}{m+M_{s-}}\left[\left(v-V_{s-}\right) \cdot n\right] n \mathbb{1}_{\left\{u \leq \frac{a_{B}\left(M_{s-}, m\right)}{m} \varphi_{B}\left(V_{s-}, v, n\right)\right\}} \\
\nu_{B}(d s, d(m, v), d n, d u) .
\end{gathered}
$$

The simulation algorithm of $(M, V)$ given in the next section might help to understand the meaning of this equation. Before giving more details, let us state the link between $(S D E)$ and $(B S)$ (see Tanaka [10] and Deaconu et al. [3] for rigorous proofs of similar results).

Proposition 2.3. Assume $(A)$. Consider a solution $\left(M_{t}, V_{t}\right)_{t \geq 0}$ to $(S D E)$. Assume that for each $t \geq 0$, the law of $V_{t}$ has a density. Thus the law $Q_{t}(d m, d v)$ of $\left(M_{t}, V_{t}\right)$ can be written as $\sum_{k>1} g(t, k, v) \delta_{k}(d m) d v$. Set $f(t, m, v)=g(t, m, v) / m$. Then $f$ is a solution to $(B S)$ in the sense of Definition 2.1.

Let us explain briefly the main idea of $(S D E)$ : we wish to build a process $\left(M_{t}, V_{t}\right)_{t \geq 0}$ whose law is the distribution of masses and velocities in the particle system. Hence, $\left(M_{t}, V_{t}\right)$ has to be the evolution of a sort of typical particle.

Recall that we are in the discrete case, so that a particle of size $m$ may be understood as being composed of $m$ atoms. We mark, at time 0 , a given atom, chosen randomly (and uniformly) among all atoms in the system. Then we denote by $\left(M_{t}, V_{t}\right)$ the mass and velocity of the particle containing our marked atom at time $t$. Such a process is naturally Markov, the mass $M$ and velocity $V$ are naturally piecewise constant, and $M$ is of course nondecreasing. Finally, Equation (2.9) explains that at some random instants (with a well-chosen rate, which appears in the indicator functions), we choose another typical particle in the system, we denote by $(m, v)$ its characteristics, and we make this particle coalesce with our typical particle (this modifies $M$ and $V$ ). Of course, we choose $(m, v)$ according to the distribution $Q_{t}=\mathcal{L}\left(M_{t}, V_{t}\right)$, since $Q_{t}$ represents the distribution of the characteristics in the system. In the same way (and independently), at some random instants (with a well-chosen rate, which appears in the indicator functions), we choose another particle in the system, we denote by $(m, v)$ its characteristics, we choose at random an impact parameter $n \in S_{2}$, and we make the particle $(m, v)$ collide with our typical particle according to $n$ (this modifies only $V)$.

Note finally that the rate $a_{S}\left(M_{s-}, m\right) / m$ is natural, since we pick in the system an atom, and denote by $m$ the mass of the particle containing this unit particle. Hence a particle of mass $m$ appears $m$ times, which leads to divide the rate $a_{S}\left(M_{s-}, m\right)$ by $m$.

Using an explicit simulation algorithm, we will prove in the next section the following result.

THEOREM 2.4. Assume $(A)$. Then there exists a solution to $(S D E)$. Furthermore, the law of $V_{t}$ has a density for each $t \geq 0$. Hence there exists a solution $f$ to (BS) (see Proposition 2.3). 
We now wish to show that under some conditions, the whole mass of the system becomes infinite as time tends to infinity. We would like to treat at least the case of cutoff hard spheres rates: $a_{S}\left(m, m_{*}\right)=a_{B}\left(m, m_{*}\right)=\left(m^{1 / 3}+m_{*}^{1 / 3}\right)$, $\varphi_{S}\left(v, v_{*}\right)=\left|v-v_{*}\right| \wedge A$, while $\varphi_{B}\left(v, v_{*}, n\right)=\left|\left(v-v_{*}\right) \cdot n\right| \wedge A$. Hence, there is an intrinsic difficulty: with the masses of the particles growing, their velocities decrease, because coalescence dissipates kinetic energy. Thus the rate of coalescence (which contains $\left.\left|v-v_{*}\right|\right)$ decreases, and hence the masses grow less and less fast. The main idea will thus be to prove that, in some sense, a particle whose mass does not tend to infinity has an energy which is bounded below.

Let us introduce an additional assumption, which says essentially that: there are not much many collisions than coalescences, and the coagulation rates do not vanish too fast.

Assumption $(B)$

1. There exists $\varepsilon>0$ such that for all $m, m_{*}$ in $\mathbb{N}^{*}$ and $v, v_{*}$ in $\mathbb{R}^{3}$,

$$
a_{S}\left(m, m_{*}\right) \geq \varepsilon a_{B}\left(m, m_{*}\right) ; \quad \varphi_{S}\left(v, v_{*}\right) \geq \varepsilon \int_{S_{2}} \varphi_{B}\left(v, v_{*}, n\right) d n .
$$

2. For all $m$ in $\mathbb{N}^{*}, a_{S}(m, m)>0$, while $\int_{\mathbb{R}^{3}} \int_{\mathbb{R}^{3}} \mathbb{1}_{\left\{\varphi_{S}\left(v, v_{*}\right)=0\right\}} d v d v_{*}=0$.

Theorem 2.5. Assume $(A)$ and $(B)$. Consider a solution $\left(M_{t}, V_{t}\right)_{t \geq 0}$ to $(S D E)$. Then $\lim _{t \rightarrow \infty} M_{t}=\infty$ a.s. As a corollary, we deduce that the corresponding solution $f(t,$.$) to (B S)$ (see Proposition (2.3)) tends to 0 in $L^{1}\left(\mathbb{N}^{*} \times \mathbb{R}^{3}\right)$, as time tends to infinity.

REMARK 2.6. The present results will be extended in [6] which is work in progress. We anticipate to obtain the corresponding results both for the discrete and continuous case and for unbounded rates. We remark, that to prove existence and uniqueness, the probabilistic arguments used here will not be sufficient.

\section{Existence and simulation}

Our aim in this section is to build explicitely a solution to $(S D E)$. We denote, for each $m \in \mathbb{N}^{*}$, by $\lambda(m)$ the maximal event rate of a particle of size $m$ :

$$
\lambda(m)=\sup _{v, m_{*}, v_{*}, n}\left[\frac{a_{S}\left(m, m_{*}\right)}{m_{*}} \varphi_{S}\left(v, v_{*}\right)+\frac{a_{B}\left(m, m_{*}\right)}{m_{*}} 4 \pi \varphi_{B}\left(v, v_{*}, n\right)\right] .
$$

Let $Q_{0}(d m, d v)$ be defined by $\sum_{k>1} k f_{0}(k, v) \delta_{k}(d m) d v$. For any $t \geq 0$, we build the following (recursive) random function.








Let $T<\infty$. Using this algorithm allows us to obtain a process $\left(M_{t}, V_{t}\right)_{t \in[0, T]}$, by stocking the successive values $\left(m_{0}, v_{0}\right),\left(m_{1}, v_{1}\right), \ldots$ of the variables $(m, v)$, the successive values $0=t_{0}<t_{1}<\ldots$ of the variable $s$, and setting $\left(M_{t}, V_{t}\right)=\sum_{i}\left(m_{i}, v_{i}\right) \mathbb{1}_{\left\{t \in\left[t_{i}, t_{i+1}\right)\right\}}$. Following the proof of [5], one may check the following result.

Proposition 3.1. Assume $(A)$, and let $T<\infty$. Then the computation of (mass, velocity) $(T)$ a.s. ends. The corresponding process $\left(M_{t}, V_{t}\right)_{t \in[0, T]}$ is a solution to $(S D E)$ on $[0, T]$.

We presented this algorithm in its simplest form here. It looks quite inefficient. One may however significantly increase its speed by using some computational tricks, see [5].

The main idea of this algorithm consists in noting that the characteristics (mass, velocity) of a typical particle are obtained by making it collide and coalesce, with wellchosen rates and acceptance-rejection procedures, with other typical particles. The characteristics of these other typical particles will be obtained by making them collide and coalesce, with well-chosen rates and acceptance-rejection procedures, the mass of other typical particles, and so on... This explains why the algorithm we propose is recursive.

To conclude the proof of Theorem 2.4, one still has to show the following lemma.

Lemma 3.1. Assume $(A)$. Consider a solution $\left(M_{t}, V_{t}\right)_{t \geq 0}$ to $(S D E)$. Then for all $t \geq 0$, the law of $V_{t}$ has a density.

Proof. First of all denote by $Q_{t}$ the law of $\left(M_{t}, V_{t}\right)$, and by $\mu_{t}(d m, d v)=$ $m^{-1} Q_{t}(d m, d v)$. Then a fair computation shows that, even if $\mu_{t}$ is not absolutely continuous, $\mu_{t}$ is a measure solution to $(B S)$ : it solves (2.8) replacing $f(s, m, v) d v$ and $f\left(s, m_{*}, v_{*}\right) d v_{*}$ by $\mu_{s}(\{m\} \times d v)$ and $\mu_{s}\left(\left\{m_{*}\right\} \times d v_{*}\right)$. See [3] for such a computation in a similar context. Denote now by $\mathcal{A}=\left\{A \in \mathcal{B}\left(\mathbb{R}^{3}\right) ; \int_{A} d v=0\right\}$. One has to show that for each $t \geq 0, m_{0} \in \mathbb{N}^{*}, A \in \mathcal{A}, \mu_{t}\left(\left\{m_{0}\right\} \times A\right)=0$. This can be done by applying the measure version of (2.8) with the function $\phi(m, v)=\mathbb{1}_{\left\{m=m_{0}\right\}} \mathbb{1}_{\{v \in A\}}$, neglecting all the loss terms, and then by applying the Gronwall Lemma to the function $\psi_{t}\left(m_{1}\right)=\sup _{m_{0} \leq m_{1}} \sup _{A \in \mathcal{A}} \mu_{t}\left(\left\{m_{0}\right\} \times A\right)$. As an example, one easily checks that for all $A \in \mathcal{A}$, all $m, m_{*}, v_{*}$, the set $B=\left\{v ; v_{* *} \in A\right\}$ still belongs to $\mathcal{A}$.

\section{Large time behavior}

Our aim in this section is to prove Theorem 2.5. In the whole section, we assume $(A),(B)$, we consider a fixed solution $\left(M_{t}, V_{t}\right)_{t \geq 0}$ to $(S D E)$. For each $t \geq 0$, we denote by $Q_{t}(d m, d v)=\sum_{k>1} k f(t, k, v) \delta_{k}(d m) d v$ the law of $\left(M_{t}, V_{t}\right)$. We know that $f$ is a solution to $(B S)$ in the sense of Definition 2.1. We begin with a lemma. 
Lemma 4.1. For any $m \in \mathbb{N}^{*}$,

$$
\int_{0}^{\infty} d t \int_{\mathbb{R}^{3}} f(t, m, v) d v \int_{\mathbb{R}^{3}} f\left(t, m, v_{*}\right) d v_{*} \varphi_{S}\left(v, v_{*}\right)<\infty .
$$

The proof is immediate, applying (2.8) with $\phi \equiv 1$, using the nonnegativity of all the involved functions, and the fact that $a_{S}(m, m)>0$ thanks to $(B)$.

Lemma 4.2. Almost surely, $M_{\infty}=\lim _{t \rightarrow \infty} M_{t} \in \mathbb{N}^{*} \cup\{\infty\}$ exists.

The proof of this is immediate, since $M$ is a nondecreasing $\mathbb{N}^{*}$-valued process. Our aim is thus to check that $P\left[M_{\infty}=\infty\right]=1$. We will assume the converse.

$\underline{\text { Assumption }(S):} P\left[M_{\infty}<\infty\right]>0$.

The key point of the proof consists in the following lemma.

Lemma 4.3. Assume $(S)$. Then there exists $t_{0} \geq 0$ and $m_{0} \in \mathbb{N}^{*}$ such that

$$
\gamma_{0}=P\left[\text { for all } t \geq t_{0}, M_{t}=m_{0} \text { and } V_{t}=V_{t_{0}}\right]>0 .
$$

Proof. We break the proof into three steps.

Step 1: First of all, the discrete nature of $M$ allows to conclude that under $(S)$, there exists $t_{1} \geq 0$ and $m_{0} \in \mathbb{N}^{*}$ such that, if

$$
\Omega_{1}=\left\{\text { for all } t \geq t_{1}, M_{t}=m_{0}\right\},
$$

then $P\left[\Omega_{1}\right]>0$. We now define

$$
\begin{gathered}
J_{t}^{S}=\int_{0}^{t} \int_{\mathbb{N}^{*}} \int_{\mathbb{R}^{3}} \int_{0}^{\infty} \mathbb{1}_{\left\{u \leq \frac{a_{S}\left(M_{s-}, m\right)}{m} \varphi_{S}\left(V_{s-}, v\right)\right\}} \nu_{S}(d s, d(m, v), d u), \\
J_{t}^{B}=\int_{0}^{t} \int_{\mathbb{N}^{*}} \int_{\mathbb{R}^{3}} \int_{S_{2}} \int_{0}^{\infty} \mathbb{1}_{\left\{u \leq \frac{a_{B}\left(M_{s-}, m\right)}{m} \varphi_{B}\left(V_{s-}, v, n\right)\right\}} \nu_{B}(d s, d(m, v), d n, d u),
\end{gathered}
$$

where $J_{t}^{S}$ (resp. $J_{t}^{B}$ ) represents the number of coalescences (resp. collisions) endured by our typical particle before $t$. With these notations,

$$
\Omega_{1}=\left\{M_{t_{1}}=m_{0}, \quad J_{\infty}^{S}-J_{t_{1}}^{S}=0\right\} .
$$

The lemma will thus be proved if there exists $t_{0} \geq t_{1}$ such that

$$
P\left[M_{t_{0}}=m_{0} ; \quad J_{\infty}^{S}-J_{t_{0}}^{S}=0 ; \quad J_{\infty}^{B}-J_{t_{0}}^{B}=0\right]>0 .
$$

Step 2: We now show that

$$
P\left[M_{t_{1}}=m_{0} ; \quad J_{\infty}^{S}-J_{t_{1}}^{S}=0 ; \quad J_{\infty}^{B}-J_{t_{1}}^{B}<\infty\right]=P\left[\Omega_{1}\right]>0 .
$$

This is not hard. Indeed, consider the successive instants of jumps $t_{1}<\tau_{1}<\tau_{2}<\ldots$ of $J_{t}^{S}+J_{t}^{B}$ after $t_{1}$, and for each $i \in \mathbb{N}^{*}$, set the random variable $Z_{i}$ to be 1 (resp. 0 ) if $\tau_{i}$ is an instant of jump of $J^{S}$ (resp. $J^{B}$ ). Then the $Z_{i}$ are independent, and thanks 
to $(B)-1$, we deduce that $P\left[Z_{i}=1\right] \geq \varepsilon^{2} /\left[1+\varepsilon^{2}\right]$. Indeed, at each instant $t$, the rate of jump $R_{t}^{S}$ (resp $R_{t}^{B}$ ) of $J_{t}^{S}$ (resp. $J_{t}^{B}$ ) is given by

$$
\begin{array}{r}
R_{t}^{S}=\sum_{m \geq 1} \int_{\mathbb{R}^{3}} a_{S}\left(M_{t-}, m\right) \varphi_{S}\left(V_{t-}, v\right) f(t, m, v) d v \\
R_{t}^{B}=\sum_{m \geq 1} \int_{\mathbb{R}^{3}} a_{B}\left(M_{t-}, m\right) \int_{S_{2}} \varphi_{B}\left(V_{t-}, v, n\right) d n f(t, m, v) d v
\end{array}
$$

so that $R_{t}^{S} \geq \varepsilon^{2} R_{t}^{B}$. Hence,

$$
P\left[J_{\infty}^{S}-J_{t_{1}}^{S}=0 ; \quad J_{\infty}^{B}-J_{t_{1}}^{B}=\infty\right] \leq P\left[\text { for all } i \geq 1, Z_{i}=0\right]=0 .
$$

One easily concludes that (4.7) holds.

Step 3: We thus deduce that on $\Omega_{1}$, the last instant of jump of $J^{B}$ is a.s. finite, so that for $t_{0} \geq t_{1}$ sufficiently large, (4.6) holds. This concludes the proof.

Lemma 4.4. Assume $(S)$. There exists a nonnegative function $\beta$ on $\mathbb{R}^{3}$ such that $\int_{\mathbb{R}^{3}} \beta(v) d v>0$ and for all $t \geq t_{0}$, all $v \in \mathbb{R}^{3}, m_{0} f\left(t, m_{0}, v\right) \geq \beta(v)$.

Proof. First of all consider the nonnegative measure $M(d v)$ on $\mathbb{R}^{3}$ defined by

$$
M(A)=P\left[\text { for all } t \geq t_{0}, M_{t}=m_{0} \text { and } V_{t}=V_{t_{0}}, V_{t_{0}} \in A\right] .
$$

This measure has a density $\beta$, since the law of $V_{t_{0}}$ has a density. This function $\beta$ is nonnegative, and thanks to Lemma $4.3, \int_{\mathbb{R}^{3}} \beta(v) d v=\gamma_{0}>0$.

Using the link between $(S D E)$ and $(B S)$, we obtain for each $t \geq t_{0}$, all $A \subset \mathbb{R}^{3}$,

$$
\int_{A} m_{0} f\left(m_{0}, t, v\right) d v=P\left[M_{t}=m_{0}, V_{t} \in A\right] \geq M(A)=\int_{A} \beta(v) d v .
$$

which ends the proof.

Proof of Theorem 2.5: Assume $(S)$. Using Lemma 4.4, we obtain for all $t \geq t_{0}$,

$$
\begin{array}{r}
\int_{\mathbb{R}^{3}} f\left(t, m_{0}, v\right) d v \int_{\mathbb{R}^{3}} d v_{*} f\left(t, m_{0}, v_{*}\right) \varphi_{S}\left(v, v_{*}\right) \\
\geq m_{0}^{-2} \int_{\mathbb{R}^{3}} \int_{\mathbb{R}^{3}} d v d v_{*} \varphi_{S}\left(v, v_{*}\right) \beta(v) \beta\left(v_{*}\right)=\delta>0,
\end{array}
$$

thanks to assumption $(B)-2$, the constant $\delta$ not depending on $t \geq t_{0}$. This contradicts Lemma 4.1 with $m=m_{0}$. Hence $(S)$ does not hold, and $M_{\infty}=\infty$ a.s. Finally, $\sum_{m>1} \int_{\mathbb{R}^{3}} f(t, m, v) d v=E\left[1 / M_{t}\right]$, which obviously tends to 0 due to the Lebesgue Theorem, since $1 / M_{t}$ is always smaller than 1 . In other words, $f(t,$.$) tends to 0$ in $L^{1}\left(\mathbb{N}^{*} \times \mathbb{R}^{3}\right)$.

\section{REFERENCES}

[1] C. Baranger, Collisions, coalescences et fragmentations des gouttelettes dans un spray: écriture précise des équations relatives au modèle TAB, mémoire de DEA, ENS de Cachan, 2001.

[2] A.V. Bobylev and R. Illner, Collision integrals for attractive potentials, J. Statist. Phys., 95:633-649, 1999.

[3] M. Deaconu, N. Fournier, and E. Tanré, A pure jump Markov process associated with Smoluchowski's coagulation equation, Ann. Probab., 30(4):1763-1796, 2002. 
[4] M. Escobedo, P. Laurençot, and S. Mischler, On a kinetic equation for coalescing particles, preprint, 2003.

[5] N. Fournier and J.S. Giet, Exact simulation of nonlinear coagulation processes, preprint 2003/11 de l'institut Elie Cartan, 2003.

[6] N. Fournier and S. Mischler, On a Boltzmann equation for elastic, inelastic and coalescing collisions, in preparation.

[7] C. Graham and S. Méléard, Stochastic particle approximations for generalized Boltzmann models and convergence estimates, Ann. Probab., 25(1):115-132, 1997.

[8] J.M. Roquejoffre and Ph. Villedieu, A kinetic model for droplet coalescence in dense sprays, Math. Models Methods Appl. Sci., 11:867-882, 2001.

[9] A.S. Sznitman, Equations de type de Boltzmann, spatialement homogènes, Z. Wahrsch. Verw. Gebiete, 66(4):559-592, 1984.

[10] H. Tanaka, Probabilistic treatment of the Boltzmann equation of Maxwellian molecules, Z. Wahrsch. Verw. Gebiete, 46(1):67-105, 1978.

[11] P. Villedieu and J. Hylkema, Une méthode particulaire aléatoire reposant sur une équation cinétique pour la simulation numérique des sprays denses, C.R.A.S. de Paris, t.325 série I:323-328, 1997. 